\title{
An experimental animal model of Kashin-Beck disease
}

\author{
GUIQING ZHANG AND JINXIAN LIU
}

From the Heilong Jian Institute of Kashin-Beck Disease, Harbin Medical College, China

SUMMARY Twelve young macaque monkeys were fed with grain and water from areas actively endemic or non-endemic for Kashin-Beck disease. Both dietary grain and water from geographical areas endemic for Kashin-Beck disease induced a sequence of pathological changes in the growth plates and articular cartilage and biochemical changes in the serum and urine of monkeys. These changes are similar to those in human Kashin-Beck disease. It is considered that this may be a simple and valuable model for the further study of this disease and its management and control. The results suggest that the pathogenetic factors of Kashin-Beck disease relate both to grain and to water in the diet in endemic areas. The experiment also shows that certain serum enzyme concentrations correlate with chondronecrosis.

Key words: chondronecrosis, osteoarthropathy.

Kashin-Beck disease (KBD) is a severe osteoarthropathy, which develops mainly in children 5-13 years of age, causing developmental disturbances, joint deformity, decrease or loss of capacity for physical labour in adulthood, and lifelong disability. The basic pathological changes are multiple areas of degeneration and necrosis of the growth plates and articular cartilages as well as proliferation or repair of cartilage secondary to chondronecrosis.

Although KBD has been studied for more than 100 years, no ideal animal model of the condition is known. The epidemiology of KBD suggests that the lesions of the disease relate both to dietary grain and to water in endemic areas. ${ }^{1}$ Necrosis of cartilage in young dogs, resembling that in human KBD, can be produced by feeding the animals with grain and water from endemic areas. ${ }^{2}$ In this paper we have attempted to reproduce an animal model of KBD in monkeys by administering a diet of grain and water from an active endemic area. The aim of this work was to test whether an animal model of KBD can be produced not only in dogs but also in monkeys, which are more closely related to man. Such an animal model of KBD should provide a method of studying the pathogenesis, prevention, and treatment of KBD.

Accepted for publication 15 June 1988.

Correspondence to Dr Guiqing Zhang. Heilong Jiang Institute of Kashin-Beck Disease, Harbin Medical College, 41 Da Zhi Street, Harbin, People's Republic of China.

\section{Materials and methods}

Twelve immature macaque monkeys (seven male and five female), aged 7-12 months and weighing approximately $1 \cdot 5-2 \mathrm{~kg}$, were obtained from the Centre for Experimental Animals in Shanghai. After receiving a standard diet for four months to adapt to the laboratory environment they were divided randomly into four groups (Table 1). Group 1 (control) received grain and water from a nonendemic area, where no children aged 6-14 years showed evidence of KBD by $x$ ray examination. Groups 2, 3, and 4 received dietary grain or water, or both, from an active endemic area, where more than $80 \%$ of phalangeal metaphyses showed radio-

Table 1 Details of the animals and their dietary treatment

\begin{tabular}{|c|c|c|c|}
\hline Group & $\begin{array}{l}\text { Number of } \\
\text { monkeys }\end{array}$ & $\begin{array}{l}\text { Average } \\
\text { weight }(k g)\end{array}$ & Grain and water \\
\hline 1 & 3 & 1.95 & $\begin{array}{l}\text { Both grain and water from } \\
\text { non-endemic area }\end{array}$ \\
\hline 2 & 3 & $2 \cdot 31$ & $\begin{array}{l}\text { Grain from non-endemic } \\
\text { area, water from endemic } \\
\text { area }\end{array}$ \\
\hline 3 & 3 & $1 \cdot 70$ & $\begin{array}{l}\text { Grain from endemic area. } \\
\text { water from non-endemic } \\
\text { area }\end{array}$ \\
\hline 4 & 3 & $1 \cdot 60$ & $\begin{array}{l}\text { Both grain and water from } \\
\text { endemic area }\end{array}$ \\
\hline
\end{tabular}


logical lesions of KBD. The grain component of the diet was corn powder $86 \%$, soya bean powder $6 \%$, yeast $2 \%$, oil $1 \%$, salt $2 \%$, calcium carbonate $2 \%$, and cod liver oil $1 \%$.

The animals were allowed to walk freely in cages and given cooked food as in a human diet. At intervals of three months until the end of the experiment the monkeys were weighed, the extremities $x$ rayed, blood samples taken for analysis of serum enzymes (lactic dehydrogenase, aspartate transaminase, alanine transaminase, alkaline phosphatase, and creatine phosphokinase), and urine samples taken for determination of creatine and creatinine. Analysis of serum enzymes was performed on MCA-III autobiochemical analytical equipment (IL Co, USA), and determination of creatine and creatinine in urine was carried out by Folin's alkaline picric acid method. Four monkeys, one from each group, were killed at seven, 13, and 17 months after the start of the special diet. The extremities of each monkey were fixed in $10 \%$ formalin, embedded in paraffin, sections $10 \mu \mathrm{m}$ thick were cut, stained with haematoxylin and eosin, and examined by light microscopy.

\section{Results}

No striking difference was observed between the control specimens of group 1 and those of the experimental groups 2,3 , and 4 as regards their general state, not even by $x$ ray examination.

\section{BIOCHEMICAL CHANGES}

Biochemical analysis of blood and urine in experimental groups 2,3 , and 4 showed increased concentrations of lactic dehydrogenase, aspartate transaminase, alanine transaminase, alkaline phosphatase, and creatine phosphokinase. Lactic dehydrogenase and alkaline phosphatase had started to change at three months but aspartate transaminase, alanine transaminase, and creatine phosphokinase not until 13 months after the start of the experiment므. (Table 2); there was an increase in urine creatine? and a decrease in urine creatinine (Table 3) as $\overrightarrow{\text { त }}$ compared with group 1 . These biochemical changes closely resemble those occurring in human KBD.

LIGHT MICROSCOPY CHANGES

At seven months after the start of the experiment no distinctive morphological changes were observed in ${ }^{\infty}$ groups 2,3 , and 4 when compared with the controle group 1 . In the animals killed at 13 and 17 months, however, marked differences in histopathology were found between the control group 1 and the othero three groups. Chondronecrosis had occurred in groups 2,3 , and 4 , similar to that found in humanoo KBD.

ARTICULAR CARTILAGE IN EXPERIMENTALO GROU PS 2,3 , A N D 4

Different degrees of chondronecrosis were found in articular cartilage in each experimental group. Ine mild cases there were small foci of individuaE chondrocyte necrosis in the deeper layers of articu栬 lar cartilage. In severe cases coagulative chon dronecrosis was not only present in the deepgion layers but also extended to the middle layer with disintegration and splitting of the necrotic tiss (Fig. 1). Chondrocytes around the necrotic lesions had formed cell clusters in an attempted repainō process.

Table 3 Creatine and creatinine in urine (at 13 months). Values are mean (SD)

\begin{tabular}{|c|c|c|}
\hline Group & Creatine (U/l) & Creatinine ( $U / l$ \\
\hline $\begin{array}{l}1 \\
2 \\
3 \\
4\end{array}$ & $\begin{array}{r}9.72(1.72) \\
15.10(1.89) \\
13.21(3.78) \\
20.74(3.77)\end{array}$ & $\begin{array}{l}87 \cdot 80(8 \cdot 13) \\
71 \cdot 54(8 \cdot 13) \\
77 \cdot 55(16 \cdot 16) \\
69 \cdot 48(5 \cdot 56)\end{array}$ \\
\hline \multicolumn{3}{|c|}{$\begin{array}{l}\text { Creatine: groups } 2,3 \text {, and } 4 p<0.05 \text {. } \\
\text { Creatinine: not significant. }\end{array}$} \\
\hline
\end{tabular}

Table 2 Comparison of activities of serum enzymes (at 13 months). Values are mean (SD)

\begin{tabular}{|c|c|c|c|c|c|}
\hline Group & $L D H^{*}(U / l)$ & $A S T^{*}(U / l)$ & $A L T^{*}(U / l)$ & $A L P^{*}(U / l)$ & $C P K^{*}(U / l)$ \\
\hline $\begin{array}{l}1 \\
2 \\
3 \\
4\end{array}$ & $\begin{array}{ll}142.6 & (16 \cdot 35) \\
147.2 & (0 \cdot 75) \\
144 \cdot 2 & (20 \cdot 50) \\
225 \cdot 2 & (18 \cdot 15)\end{array}$ & $\begin{array}{l}37.9(3.10) \\
39.45(1.65) \\
46.45(6.25) \\
88.90(18.30)\end{array}$ & $\begin{array}{l}40 \cdot 90(0.60) \\
42 \cdot 84(4.40) \\
58 \cdot 70(3.50) \\
99 \cdot 30(4 \cdot 29)\end{array}$ & $\begin{array}{l}378.6(115 \cdot 13) \\
470.1(26 \cdot 20) \\
469.15(39.85) \\
464.5(135.5)\end{array}$ & $\begin{array}{ll}61.15 & (5.00) \\
57.25 & (12.45) \\
94.50 & (69.709 \\
203.5 & (64.45)\end{array}$ \\
\hline
\end{tabular}

${ }^{*} \mathrm{LDH}=$ lactic dehydrogenase; $\mathrm{AST}=$ aspartate transaminase; $\mathrm{ALT}=$ alanine transaminase; $A \mathrm{AP}=$ alkaline phosphatase; $\mathrm{CPK}=$ creatine phosphokinase.

LDH: group 4 p $<0.01$

AST: group $3 p<0.05$, group $4 p<0.01$

ALT: group $3 \mathrm{p}<0.05$, group $4 \mathrm{p}<0.01$.

CPK: group $3 \mathrm{p}<0.05$, group $4 \mathrm{p}<0.01$. 
GROWTH PLATE

Chondrocytes were reduced in number and size, suggesting low proliferative activity and some disturbance of maturation. In addition, there was a distinctive zonal necrosis. This had a coagulative character and was present on the metaphyseal side of the growth plate. Granulation tissue arising from the marrow space was frequently present close to these nectrotic foci (Fig. 2).

Several of these foci in different stages of necrosis

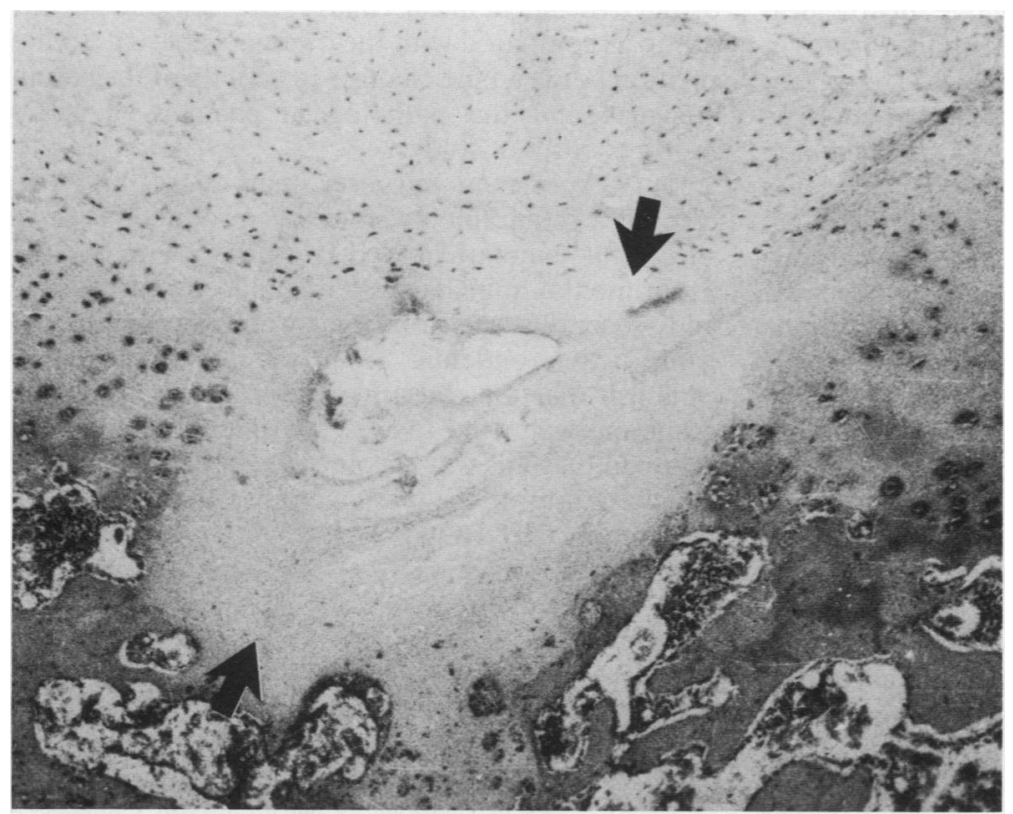

Fig. 1 Articular cartilage, showing a large necrotic focus (arrows) with cavity and proliferative cells (grain group 3).

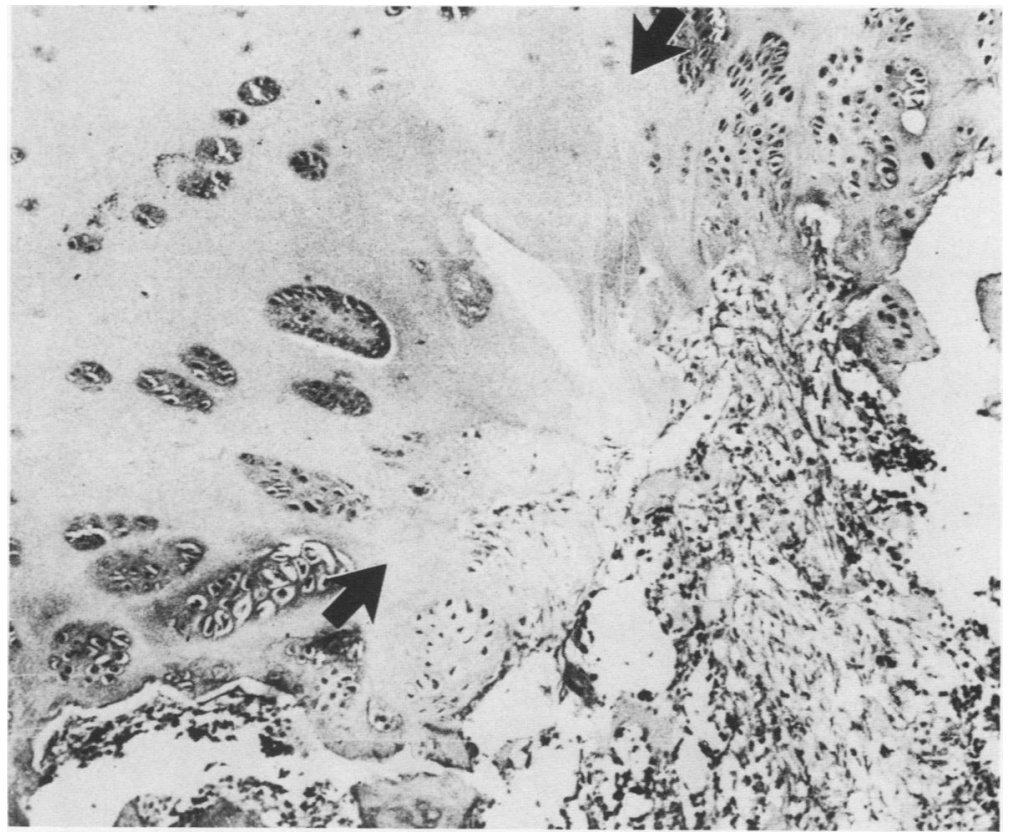

Fig. 2 Epiphyseal plate, showing a small necrotic focus (arrows) with fissure (grain and water group 4). 
(old and new lesions) quite often occurred in the same specimen in both articular cartilage and growth plate.

\section{Discussion}

It is well known that the distinctive histological lesion ${ }^{4}$ of KBD is a zonal necrosis of chondrocytes in the growth plate and articular cartilage. The necrosis is of the coagulative type with a characteristic distribution starting at the base of the cartilage and extending from there to the superficial layer and may involve the whole thickness of the cartilage. Clusters of chondrocytes form round the necrotic foci, disintegration and absorption of necrotic material being followed by invasion from the bone marrow space, leading to repair of the necrotic cartilage and reconstruction of new bone secondary to chondronecrosis. New and old lesions often coexist.

The biochemical features of patients with $\mathrm{KBD}^{3}$ include a rise of activity of lactic dehydrogenase, aspartate transaminase, alanine transaminase, alkaline phosphatase, and creatinine phosphokinase in the serum, while in the urine there is an increase in creatine and a decrease in creatinine. High activities of lactic dehydrogenase, aspartate transaminase, alanine transaminase, alkaline phosphatase, and creatine phosphokinase and marked chondronecrosis occurred simultaneously in experimental groups 3 and 4 in this study. Alkaline phosphatase concentrations in KBD may reflect an abnormality of calcification in cartilage. It appears that pathological calcification may follow chondronecrosis. The increase in creatine and the decrease in creatinine may? be associated with injury to muscle. These biochemical changes correlated with the morphological abnormalities in groups 3 and 4 . Although chondronecrosis occurred in experimental group 2, no $\overline{\bar{n}}$ distinctive biochemical changes were found in this $\vec{\infty}$ group as compared with the others (Table 2). So the relation between the changes in activity of the serumes enzymes and the pathological changes of $\mathrm{KBD} \vec{\circ}$ requires further study.

Our experience suggests that there are two $\vec{\omega}$ essential requirements for the production of ao successful animal model of KBD. Firstly, the experimental animals chosen should be susceptible and, secondly, the grain and water given must beiv from active endemic areas. It is important to $\vec{p}$ establish that the causative agent is actually in the grain and water fed to the experimental animals. It is hoped that this will become a useful animal mode $L$ for the study of the pathogenesis, treatment, and prevention of $\mathrm{KBD}$.

\section{References}

1 Lianqun L. Study of relation between grain, water and Kash Beck disease. Compilation of Kashin-Beck disease. 1979: 1-17.

2 Guiqin Zhang. The experimental study of animal model of Kashin-Beck disease. Heilong Jiang Medicine 1981: 11.

3 Tongxu Y. The development of biochemistry in Kashin-Beck disease. Yongsho investigation of collection of Kashin-Beck $\mathbb{D}$ disease. 1984: 275-86.

4 Donxi M O. Pathology of Kashin-Beck disease. Chinese $\overline{\widehat{O}}$ Medical Encyclopedia 1985: 22. 